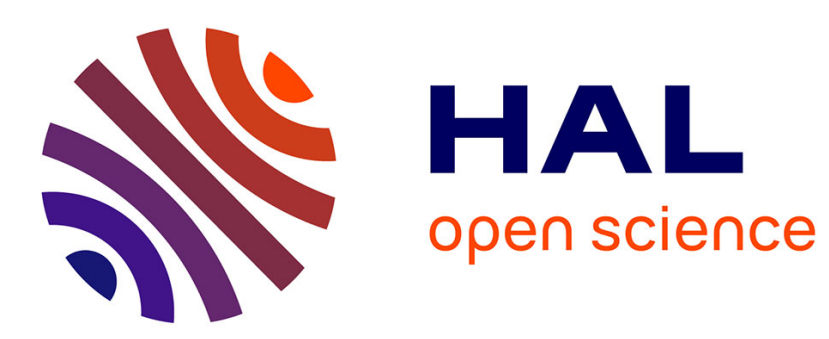

\title{
Musée et histoire de l'immigration, un enjeu pour toutes les nations
}

\author{
Vincent Viet
}

\section{To cite this version:}

Vincent Viet. Musée et histoire de l'immigration, un enjeu pour toutes les nations. Hommes \& migrations, 2005, Les chantiers de l'histoire. Historiographie de l'immigration., 1255, pp.72-78. hal03525120

\section{HAL Id: hal-03525120 \\ https://hal.science/hal-03525120}

Submitted on 28 Jan 2022

HAL is a multi-disciplinary open access archive for the deposit and dissemination of scientific research documents, whether they are published or not. The documents may come from teaching and research institutions in France or abroad, or from public or private research centers.
L'archive ouverte pluridisciplinaire HAL, est destinée au dépôt et à la diffusion de documents scientifiques de niveau recherche, publiés ou non, émanant des établissements d'enseignement et de recherche français ou étrangers, des laboratoires publics ou privés. 


\section{Musée et histoire de l'immigration, un enjeu pour toutes les nations}

\section{Vincent Viet}

\section{Résumé}

"Récente", selon les uns, ou "faussement récente", selon les autres, l'histoire de l'immigration a véritablement pris son essor, pour la plupart des pays européens, dans les années quatre-vingt, alors qu'il existait déjà, dans ce domaine, des travaux de démographie et de sociologie. Elle a rapidement été confrontée à la multitude des approches possibles et à la difficulté des historiens d'imposer leur spécificité face à une hybridation des disciplines. Dans ce contexte, quels sont les enjeux, les risques et les apports, d'une Cité nationale de l'histoire de l'immigration ?

\section{Citer ce document / Cite this document :}

Viet Vincent. Musée et histoire de l'immigration, un enjeu pour toutes les nations. In: Hommes et Migrations, $\mathrm{n}^{\circ} 1255$, Mai-juin 2005. Les chantiers de l'histoire. Historiographie de l'immigration. pp. 72-78;

doi : https://doi.org/10.3406/homig.2005.4352

https://www.persee.fr/doc/homig_1142-852x_2005_num_1255_1_4352

Fichier pdf généré le 27/02/2019 


\section{Musée et histoire de l'immigration, un enjeu pour toutes les nations}

"Récente", selon les uns. ou "faussement récente". selon les autres. l'histoire de l'immigration a véritablement pris son essor, pour la plupart des pays européens. dans les années quatre-vingt. alors quil existait déjà. dans ce domaine. des travaux de démographie et de sociologie.

Elle a rapidement été confrontée à la multitude des approches possibles et à la difficulté des historiens dimposer leur spécificité face à une hybridation des disciplines. Dans ce contexte. quels sont les enjeux. les risques et les apports. d'une Cité nationale de l'histoire de limmigration?

par Vincent Viet,

historien,

chargé de mission

à la MiRe-DREES

1)- Voir article

de Gérard Noiriel,

"Histoire de l'immigration en France. Etat des lieux, perspectives d'avenir", dans ce numéro.

\section{2)- Voir article de David} Feldman, "Immigrés et historiographie au Royaume-Uni", dans ce numéro.

3)- Voir article de Rainer Ohliger, "L'histoire de l'immigration en Allemagne : des défis historiographiques et culturels", dans ce numéro.
Dès le milieu des années vingt, les Etats-Unis avaient ouvert la voie, en développant les premières études sur l'histoire de l'immigration. Celle-ci s'est affirmée, dans les années quatre-vingt-dix, comme "domaine de recherche historique" à part entière. On se trouve depuis lors en présence d'un champ en constante évolution qui a connu, selon Gérard Noiriel(1), trois générations successives de chercheurs. Mais on peut dire aussi, et c'est une autre façon de voir les choses, que l'histoire de l'immigration est un "sous-produit" de l'éclatement de l'histoire dans les années quatre-vingt et, plus particulièrement, de l'histoire sociale qui s'est longtemps identifiée à l'histoire du mouvement ouvrier. Ce n'est certes pas un hasard si, comme l'ont rappelé David Feldman ${ }^{(2)}$ et Rainer Ohliger( ${ }^{(3)}$, l'histoire de l'immigration en France, en Grande-Bretagne et en Allemagne est souvent vue "d'en bas". Cette histoire est devenue, comme d'ailleurs l'histoire culturelle ou l'histoire des femmes, une histoire "totale", c'est-à-dire soucieuse et capable de mobiliser tous les registres d'explication disponibles (démographique, politique, économique, culturel, social, administratif, juridique, etc.).

D'autre part, la diversification des angles d'approche et l'hybridation des disciplines sont bien sûr concomitantes. Inutile d'insister sur le premier point ! L'éclatement de l'histoire sociale, son ouverture sur l'histoire culturelle et le développement de la microhistoire ont amené les chercheurs à travailler sur les quartiers, les catégories socioprofessionnelles (commerçants, étudiants, etc.), les cultures de l'immigration, les trajectoires et réseaux des migrants, le genre, etc. Quant à l'hybridation, elle est attestée - la plupart des intervenants l'ont souligné - par l'ouverture de l'histoire sur les 
sciences sociales, en particulier sur la sociologie. Mais ce mouvement est loin d'être à sens unique : les sciences sociales se nourrissent en retour des apports de l'histoire et empruntent aux historiens leurs propres méthodes en recourant aux archives écrites, sonores et audiovisuelles. De là une vraie difficulté pour ces derniers à affirmer leur spécificité, à asseoir leurs compétences et à institutionnaliser leur domaine de recherche.

Conséquence de ce dynamisme intellectuel, des postulats ou des présupposés inhérents aux recherches pionnières des années quatrevingt sont désormais remis en cause. C'est que les hypothèses s'affinent, les catégories d'analyse se précisent, les vérifications se multiplient. Aristide Zolberg ${ }^{(4)}$ et Catherine Collomp ${ }^{(5)}$ nous en ont donné une nouvelle preuve dans ce colloque en bousculant bien des idées reçues sur le cas des Etats-Unis. Le premier a ainsi récusé l'image d'une nation américaine qui aurait accueilli continûment et régulièrement des migrants de toutes les nations. La seconde a montré combien les travaux sur l'ethnicité et la culture des migrants avaient remis en cause, dans les années quatre-vingt, la notion de l'immigrant "déraciné".
4)- Voir article d'Aristide Zolberg, "Trois siècles d'immigration aux

États-Unis", dans ce numéro.

5) Voir article de Catherine Collomp, "Histoire de l'immigration aux Etats-Unis, un bref état de la question", dans ce numéro.

Intérieur du palais de la Porte Dorée. 


\section{Les lacunes de l'histoire de l'immigration}

Il n'empêche ! D'importantes lacunes subsistent, très variables d'un pays à l'autre. Certaines d'entre elles s'expliquent par les modes de structuration et de financement des milieux scientifiques ou par les cloisonnements des spécialités historiques. Si les migrations religieuses de l'époque moderne (les huguenots français) vers l'Allemagne ont fait l'objet de nombreux travaux, c'est parce qu'il existe, dans ce domaine, une solide tradition de recherche et un milieu d'historiens depuis longtemps constitué. La même observation vaut pour les Pays-Bas, où les études sur l'émigration apparaissent très concentrées sur les États-Unis, alors que

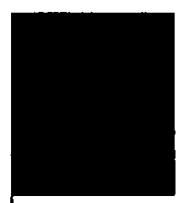

Le rayonnement politique, culturel et économique d'un pays passe aussi par l'immigration et par sa capacité à valoriser son passé de grand pays d'immigration. l'émigration des Hollandais a été très importante vers les colonies de 1600 à 1950 , et vers l'Allemagne de 1870 à 1950. On voit bien, enfin, que la spécialisation des historiens par période ou par domaine de recherche créée des angles morts, voire des apories. C'est ainsi que les migrations forcées de main-d'œuvre vers l'Allemagne durant les deux guerres mondiales ne sont pas véritablement abordées par les historiens de l'immigration qui travaillent sur le temps long ; elles sont certes analysées par les historiens de la Première Guerre mondiale et par ceux de la Seconde, mais sur le temps court et selon des grilles d'interprétation bien spécifiques.

Au-delà de ces tendances communes, il semble nécessaire d'introduire au moins deux sérieuses nuances.

Il est clair, tout d'abord, que les histoires nationales pèsent encore sur la construction des objets. On le voit bien s'agissant du lien entre immigration et passé colonial. Ce lien n'a guère de sens - ou un sens très limité - dans un pays comme l'Allemagne qui a perdu ses colonies en 1918, alors qu'il revêt un sens aigu - quoique diversement connoté et insuffisamment construit - en France, en Grande-Bretagne et aux Pays-Bas. En outre les mythes nationaux fondateurs ou les imaginaires collectifs structurent toujours des conceptions différentes de l'intégration. Non sans surprise d'ailleurs! Aux États-Unis, "'incorporation communautaire", selon Aristide Zolberg, apparait rétrospectivement compatible avec la survalorisation de la réussite individuelle ; en France, l'intégration individuelle dans une société ostensiblement laïque s'est accommodée de certaines formes d'intégration communautaire, qui ont été sous-estimées par les historiens.

Enfin et surtout, les pays d'immigration n'ont pas le même rapport aux phénomènes migratoires que les pays soit d'émigration, soit d'émigration et d'immigration. Si la problématique de l'immigration semble naturellement s'imposer en France, celle de l'émigration ou des migra- 
tions internes tient une place au moins aussi importante en Allemagne. C'est vrai aussi des pays - d'émigration - ayant connu leur transition démocratique plus tardivement qu'en France. Il serait, par ailleurs, intéressant de voir dans quelle mesure la transformation de certains pays d'émigration en pays d'immigration (cas de l'Espagne, du Portugal et de l'Italie) modifie les représentations nationales et notamment les interactions entre l'identité nationale et les phénomènes migratoires.

\section{L'apport d'une Cité nationale de l'histoire de l'immigration?}

Tout d'abord une clarification des concepts. Les concepts les plus questionnés et les plus débattus au cours de la première journée du colloque ont été "l'émigration", "l'immigration" et les "migrations internes". Il existe à cet égard des définitions communément admises par les historiens, mais celles-ci ont du mal à s'imprimer dans les consciences et des efforts de pédagogie s'avèrent nécessaires. Rien ne dit que ces définitions résisteront aux changements survenus dans la nature des flux migratoires. On assiste, en effet, dans la plupart des Etats ayant accueilli plusieurs générations d'immigrants, à une stratification des figures du migrant : l'immigré (ou migrant ayant franchi la frontière d'un État pour s'y installer) coexiste avec le migrant (présent à titre transitoire dans un État), les enfants d'origine étrangère et le "migré" (c'est-à-dire, en fin de course, celui qui est passé d'un pays à l'autre et a fini par échouer dans un pays étranger où il terminera ses jours). Enfin, il n'est pas sûr que le franchissement d'une frontière d'État suffise à caractériser "l'immigré". Comme l'a fort bien souligné David Feldman, il existe bien d'autres frontières : locales, juridiques (y compris la frontière de la nationalité), dans l'exercice des droits ou encore dans l'accès aux droits, etc.

On peut en attendre, en second lieu, une meilleure connaissance des ressorts de l'immigration et de l'émigration. Les travaux historiques sur l'immigration croisant les données relatives aux pays de départ et aux pays d'arrivée sont en fait très rares. Or, la création d'un lieu de mémoire comme la Cité devrait permettre, notamment à l'occasion d'expositions croisées, de réunir toutes ces données (les deux faces d'un même symbole), de mieux cerner les motivations et les trajectoires des migrants ; en bref, de comprendre les ressorts de l'émigration et de l'immigration et poser ainsi le "regard sur les deux rives", comme le dit Abdelmalek Sayad $^{(6)}$. C'est, en fait, toujours la question des origines qui se trouve posée : pourquoi mes ascendants ont-ils quitté leur terre d'origine ? Comment le pays qui les accucillit est-il devenu mon propre pays ?

Meilleure connaissance des ressorts de l'immigration et de l'émigration, mais aussi meilleure connaissance des méthodes et des politiques d'intégration dans une optique clairement comparatiste. Danièle Joly(7) a rappelé que la Grande-Bretagne s'était engagée, au cours des années
6)- Abdelmalek Sayad, La double absence, Paris, Seuil, 1999.

7)- Voir article de Danièle Joly, "La gouvernance de l'immigration et de l'intégration", dans ce numéro. 
tions migratoires, l'analyse en termes de diaspora ou de réseaux révèlent des situations bien plus complexes ou enchevêtrées.

Le troisième, corrélé aux deux autres, serait de figer les représentations au nom d'une conception passéiste de l'histoire, aux dépens du lien - toujours précieux pour la mémoire - entre le passé et le présent. S'il est trop déphasé par rapport à la réalité du présent ou s'il ne rend pas compte de la diversité actuelle des formes de migrations, le "message" historique peut perdre une grande part de son intelligibilité.

Alors comment conjurer ce triple risque ? En introduisant du transversal, du transnational et de l'international.

Une telle posture oblige à compléter l'analyse de l'apport de l'immigration à la France par la valorisation des transferts de culture, de savoir-faire, d'hybridation des cultures entre la France, pays d'immigration, et les pays d'émigration, y compris les anciennes colonies françaises. Il s'agit de faire passer l'idée que le rayonnement politique, culturel et économique d'un pays passe aussi par l'immigration et par sa capacité à valoriser son passé de grand pays d'immigration.

Elle implique également de se montrer attentif aux différentes catégories du droit. Dans les discussions qui ont eu lieu pendant la première journée, la notion de "respect" a été à plusieurs reprises évoquée. Or le respect se construit - entre autres choses - à travers la reconnaissance de droits attachés à la personne du migrant. Les juristes en ont tôt pris conscience, dès la constitution des États nation, à travers le "droit des gens" qui comportait, au début du $\mathrm{XX}^{\mathrm{e}}$ siècle, deux composantes : le droit international public qui concernait les rapports des États entre eux et le droit internat privé qui régissait les relations entre personnes appartenant à des Etats différents. Létude socio-historique comparée du droit international privé permettrait de comprendre comment ont été résolus les conflits entre les lois et le statut personnel des migrants. Il serait non moins intéressant de voir dans quelle mesure le droit civil des Etats-nation s'est montré conciliant à l'égard des migrants qui contribuaient à enrichir les économies nationales.

Il faut enfin tenir compte des approches nouvelles qui bousculent les cadres de pensée traditionnels de l'immigration et de l'immigration. Les analyses en termes de circulations migratoires en font partie, qui bousculent les cadres de pensée traditionnels de l'immigration ou de l'émigration. Elles font, en effet, ressortir le décalage de plus en plus net entre la volatilité et la versatilité de certains phénomènes migratoires et la gestion encore très nationale, très régalienne des questions migratoires par les États. Il est important que ces analyses aient droit de cité dans la nouvelle Cité si on veut que celle-ci s'ancre résolument dans le présent.

Je terminerai sur la notion d"universalisme français" de Théodore Zeldin $^{(8)}$. C'est vrai qu'il existe un certain universalisme français ou, à
8)- Theodore Zeldin, Histoire des passions françaises:

1848-1945 (Histoire de France), Paris, Payot, 2003. 
tout le moins, une illusion universaliste, qui ne demande qu'à s'exprimer. Mais cet universalisme est indissociable, comme d'ailleurs son homologue américain, d'un certain ethnocentrisme. Il s'incarne, s'agissant du cas français, dans le sentiment d'appartenir, depuis la Révolution française, à une collectivité forgée par l'histoire et les expériences vécues en commun, autrement dit la Nation. Or, c'est bien ce sentiment sous sa forme républicaine qui structure le projet de la Mission de préfiguration. Il s'agit tout à la fois d'aller au-delà du sens national de l'immigration en montrant que celle-ci a une résonance universelle, et de montrer que l'immigration est constitutive de notre identité nationale, comme le suggère la formule, "leur histoire est notre histoire". Ce qui revient à élargir la conception classiquement française de la Nation, celle d'Ernest Renan, qui niait les différences au nom d'une histoire commune, en reconnaissant explicitement la contribution des étrangers à cette histoire. La dette est double : envers notre histoire qui n'a pas su intégrer dans son récit cette contribution et envers les étrangers, c'est-à-dire nous-mêmes. 